\title{
Tolowa Peak Pitch Alignment: Perspectives from an Autosegmental-Metrical Framework ${ }^{1}$
}

\author{
CHRISTOPHER S. DOTY and SUSAN G. GUION \\ University of Oregon
}

\section{Overview}

The preliminary results of an investigation into the alignment of lexical pitch accents in Tolowa (Athabaskan) within an autosegmental-metrical framework are reported. Of specific interest were the peaks of prominent rising-falling pitch contours which are found to occur on some stressed syllables, and how the peaks of those contours are aligned within the syllable. Further, potential effects of segmental structure on the alignment of the peak were investigated. We begin with a brief introduction to Tolowa prosody and peak pitch-accent alignment before turning to the results of the study.

\subsection{Background on Tolowa}

Tolowa is an Athabaskan language spoken in an area straddling the OregonCalifornia border along the Pacific coast. Only three speakers of the language remain, although revitalization efforts are currently underway.

Despite being a member of the Pacific Coast Group of the Athabaskan family - a group of languages which have traditionally been thought of as nontonal-Tolowa was first described as having pitch accent, which was realized as high pitch on various syllables (Bright 1964). Subsequent work, however, seemed to indicate that Tolowa was perhaps better thought of as having stress accent, and

\footnotetext{
1 Our thanks go out to Smith River Rancheria, especially Loren Me'-lash-ne Bommelyn, Eunice Xash-wee-tes-na Bommelyn, Margaret Dee-'ishlh-ne Brooks, and Marva Sii -xuu-tes-na Scott, for sharing their language with us and for their assistance, support and patience on this project, as well as for allowing us to have access to their immense collection of recordings of their language. Although we were only fortunate enough to meet one of the speakers used in the analysis (Loren Me'-lash-ne Bommelyn), we are nonetheless indebted to the other speakers used in the study for sharing their language with the linguists they worked with: Sam K'ay-lish Lopez, Ed "Gobel" Lha'-dvn-tes-na Richards, Amelia Yuu-k'wvt-day-na Brown, Harriet Sa'lh-'vs Smith, and one other speaker previously recorded by Mary Woodward. Shu' shaa naa-ghu'-la. We would also like to thank Janne Underriner, Gwendolyn Lowes, and Melissa Redford for their helpful comments on this project. Errors, of course, remain our own.
} 


\section{Christopher S. Doty and Susan G. Guion}

the high pitch present on some syllables was simply an acoustic correlate of this stress (Tuttle 1990).

Although it appears that Bright and Tuttle were describing the same phenomenon, as their analyses often agree about where high pitch/stress occurs, it seems that each may have been considering only one of the phenomena actually present in Tolowa. Our examination of stressed syllables indicated that there are two distinct types of Bright's "high" pitch accent which can occur: 1) a high, level pitch which is maintained for the entire voiced portion of the syllable or 2) a rising-falling pitch accent (henceforth "contour pitch accent") which peaks at about mid-syllable, and then falls to the end of the syllable. Example F0 contours for each of the two types of pitch accent can be found in Figure (1).

(1) Pitch tracks for see [se:] 'stone' (top, high flat pitch accent) and sree [se:] 'sap' (bottom, contour pitch accent) from speaker AB.

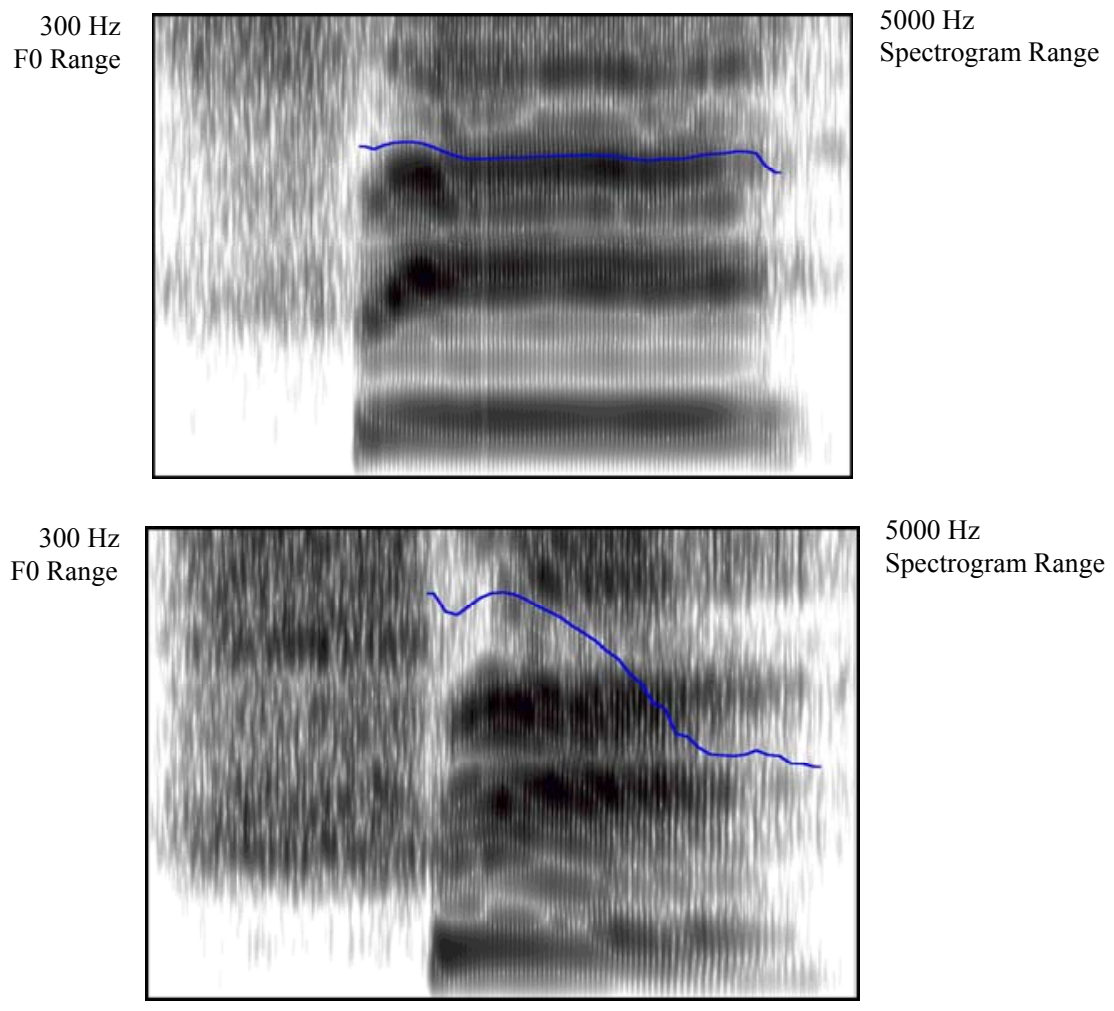

Furthermore, a preliminary analysis of unstressed syllables indicated that they do not carry pitch accent. Given this, it appears that both stress and pitch accent may be relevant for a full description of Tolowa prosody. However, the present study was not focused on the realization of stress, but instead sought to examine the alignment of the peaks of the contour pitch accent in stressed syllables. 


\section{Tolowa Peak Pitch Alignment}

\subsection{Background on Peak Pitch Alignment}

In recent years, many researchers have examined the ways in which pitch accent peaks and valleys align with the segmental string. Much of the work in this area has been conducted within an autosegmental-metrical framework. This school (described thoroughly in Ladd 1996) asserts that rises and falls in pitch are not themselves targets, but are simply the result of transitions between various level pitch targets (that is, the target itself occurs at a specific height). It is these targets that are the primary unit of pitch contours, and any movement in pitch is simply the result of linking two targets together across a given temporal distance. The utility of this view is that it allows what at first appear to be highly variable pitch contours to be shown to be very precisely aligned with a given point in the segmental string when various effects, such as the temporal distance between the targets and the segmental structure at the location of the alignment, are taken into account. Of the many factors which have been shown to effect the alignment of the peak, two specific considerations will be addressed for the Tolowa data: 1) whether the domain of alignment is the rhyme or syllable and 2) what the effect of segmental structure on that alignment might be. Although previous work within this framework has examined the alignment of both high and low targets, only the alignment of the high will be discussed here as it is most relevant to the examination of Tolowa which follows.

In one of the first studies to specifically address the alignment of peak pitch accent, Silverman and Pierrehumbert (1990) found that, in a model designed to predict the alignment of high targets in English, the proportion of the rhyme had more predictive power than a proportion calculated from the duration of the entire syllable. Further, they demonstrated that a proportion served as a better modeling tool than raw duration. Arvaniti, Ladd and Mennen (1998) present a similar result for Modern Greek. They found that the alignment of the peak of the high target of a given pitch accent occurs just after the onset of the following syllable's vowel, and thus seems to be aligned with the rhyme (albeit of a different syllable).

However, studies on other languages have found that the raw duration of the entire syllable is best able to describe the alignment of the pitch peaks. Prieto, Santen and Hirschberg (1995) found that, when potential confounds such as stress clash are carefully controlled, the duration of the syllable provides the best description of peak pitch alignment in Mexican Spanish, rather than a proportion of either the entire syllable or of only the rhyme.

In addition to addressing the issue of syllable or rhyme, some studies have also examined the effect that different segmental contexts can have on the alignment of the peak. Arvaniti et al. (1998) found that, in situations of peak delay (i.e., where the high peak of a pitch accent is actually aligned just after the beginning of the syllable following the accented syllable), following syllables that begin with nasals saw an earlier alignment of the peak, while those with fricative onsets saw a relatively later peak, though both occurred during the following syllable's vowel. Stops patterned with either one or the other, depending on speaker. These differences were attributed to the fact that the nasals were shorter 


\section{Christopher S. Doty and Susan G. Guion}

than the fricatives (although it is not clear if the difference in stop patterning by speaker can be explained by the length of the segments).

Based on this background of peak pitch alignment in other languages, two research questions were posed for Tolowa in order to determine to what extent peak pitch alignment in an Athabaskan language is similar to or different from what has been seen in Indo-European languages. First, in what way does the peak of the contour pitch accent align with the segmental string, and is the rhyme or the entire syllable the domain for the alignment of the peak? Second, how does the segmental structure of the syllable affect the alignment of the peak?

\section{Method}

\subsection{Selection of Tokens}

Three types of stressed syllables with contour pitch accents were selected for analysis. The first type consists of open syllables with long vowels (CVV). All word-internal open syllables contain long vowels in Tolowa. Oral and nasalized vowels were grouped together in the analysis, as none of the statistical tests which were used indicated that there was a significant difference between the two vowel types. The second syllable type considered is closed by a nasal (CVN). Finally, syllables closed by a voiceless obstruent (CVO) were considered. In Tolowa, the only obstruents allowed syllable finally are voiceless fricatives or aspirated stops.

The words analyzed were selected from recordings made by several linguists beginning in the 1950s. Five speakers were selected for analysis based on the number of overall words produced on the recordings as well as the quality of the recordings in order to ensure that accurate pitch tracks could be obtained. One additional, living speaker-Loren Me'-lash-ne Bommelyn, who has been extremely active in preserving Tolowa culture and language for more than thirty years and who has an M.A. in linguistics - was also recorded for inclusion in the analysis, for a total of six speakers.

Because the words were selected from a variety of old recordings, it was not possible to match words across all six speakers. Thus, words were simply sorted into the three syllable types (CVV, CVN, CVO). A set of example tokens for each speaker is presented in Table (1) below in both the IPA and the tribal orthography presented in Bommelyn (2006). A total of 217 words (76 CVV, $33 \mathrm{CVN}$ and 108 $\mathrm{CVO})$ were used for the present analysis.

In cases where speakers produced more than one repetition of the word, the first clear repetition was used. All words were pronounced in isolation, except for the productions by the living speaker, LB, which were collected in the carrier phrase Dii-dvn xee-naa-ghitlh-yash ['ti.tın ,xe:.na:.'yitt.jaf] 'Now we are saying'. 
(1) List of sample words for each syllable type from each speaker. Boldface indicates the stressed, contour pitch-accented syllable used in the analysis.

\begin{tabular}{|c|c|c|c|c|c|c|}
\hline & \multicolumn{2}{|c|}{ CVV } & \multicolumn{2}{|c|}{ CVN } & \multicolumn{2}{|c|}{$\underline{\text { CVO }}$} \\
\hline & IPA & Tribal & IPA & Tribal & IPA & Tribal \\
\hline \multirow{2}{*}{ SL } & mis.ne? & mii-ne' & nan. Ts'sn & nan-ts'vn & ka?.sıs & ga'-srvsr \\
\hline & \multicolumn{2}{|c|}{ 'back' } & \multicolumn{2}{|c|}{ 'mountain' } & \multicolumn{2}{|c|}{ 'crab' } \\
\hline \multirow{2}{*}{ HS } & tu:.ma & duu-ma & $\mathrm{k}^{\mathrm{w}}, \Delta \mathrm{n} \cdot \int \tilde{\mathrm{a}}$ & k'wvn-sha & $\operatorname{tuk}^{\mathrm{h}}$ & lhuk \\
\hline & \multicolumn{2}{|c|}{ 'sea anemone' } & \multicolumn{2}{|c|}{ 'raccoon' } & \multicolumn{2}{|c|}{ 'salmon/fish' } \\
\hline \multirow{2}{*}{ ER } & mer.ne? & mee-ne' & $\min$ & Ihnin & $\mathrm{met}^{\mathrm{h}}$ & met \\
\hline & \multicolumn{2}{|c|}{ 'house' } & \multicolumn{2}{|c|}{ 'raw' } & \multicolumn{2}{|c|}{ 'stomach' } \\
\hline \multirow{2}{*}{ LB } & ka:.si & gaa-si & $\int \Lambda \mathrm{m}$ & shvm & k'a:.t'ss & k'aa-t'vsr \\
\hline & \multicolumn{2}{|c|}{ 'acorn straining basket' } & \multicolumn{2}{|c|}{ 'good' } & \multicolumn{2}{|c|}{ 'ant' } \\
\hline \multirow{2}{*}{ MW } & se: & sree & man. $\overline{t f a} \mathrm{a}$ & man-cha' & SAS & srvsr \\
\hline & \multicolumn{2}{|c|}{ '(tree) sap' } & \multicolumn{2}{|c|}{ '(white person) dress' } & \multicolumn{2}{|c|}{ 'wood' } \\
\hline \multirow{2}{*}{$\mathbf{A B}$} & $\chi \mathrm{i}:$ & xii & Swan & srwvn & 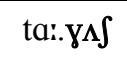 & daa-ghvsh \\
\hline & 'spr & tree' & \multicolumn{2}{|c|}{ 'dirty' } & \multicolumn{2}{|c|}{ 'snake' } \\
\hline
\end{tabular}

\subsection{Procedure \& Measurements}

Syllables were isolated in Praat (Boersma and Weenik 2006), and the duration of the rhyme and of the entire syllable were measured, as well as the duration (in ms) from the onset of both the syllable and rhyme to the temporal point at which the peak occurred. The height of the peak (in $\mathrm{Hz}$ ) was also measured, as was the height of the low at vowel onset. Vowel onset was chosen as the location to measure the low due to the fact that many of the tokens in the current data set had voiceless onset consonants, making any measurement before vowel onset impossible. For the sake of consistency, then, F0 was measured at vowel onset, even when the initial consonant was voiced and the low occurred before the onset of the vowel. In cases where F0 fell slightly after vowel onset before rising to the peak, however, the actual low was measured.

Duration of the rhyme was measured from the beginning of the vowel through the end of the syllable. The onset of the vowel as measured at the zero-crossing of the first clearly-repeating voicing cycle that coincided with the onset of second formant (F2) energy. Duration of the syllable was measured from the beginning of the onset consonant through the end of the syllable.

In order to determine syllable offset in a consistent manner, the following criteria were used for each syllable type. For CVV syllables, the end of the rhyme was taken to be the end of clear F2 energy that coincided with the end of voicing. For CVN syllables, the end of the nasal was considered to be the end of voicing in the case that the nasal was followed by a voiceless consonant or was word-final. If the nasal was followed by a voiced segment, offset was placed at the abrupt change in amplitude and formant structure between the nasal and the following segment. For CVO syllables closed with fricatives, offset was placed at the end of 


\section{Christopher S. Doty and Susan G. Guion}

the high-frequency aperiodic noise associated with the fricative. In CVO syllables closed by stops, syllable offset was placed at the end of the burst and aspiration of the stop release. Because syllable-final stops are uniformly aspirated in Tolowa, this always included a period of aspiration.

\section{Results and Discussion}

\subsection{Domain of Peak Pitch Alignment}

Before presenting the results, it should be noted that the peak in both CVN and CVO syllables was seen to occur just slightly before the onset of the coda consonant. With CVV syllables, there was no such pattern, with the peak occurring in a seemingly arbitrary position during the vowel.

Based on the measurements taken, two proportions were computed for each token: one with the peak location calculated as a percentage of the duration of the syllable, and one with peak location calculated as a percentage of the rhyme. Means of the two proportions of peak location for both syllable and rhyme are presented in Tables (2) and (3), respectively.

(2) Results for peak location as a proportion of syllable duration.

\begin{tabular}{cccccccc}
\hline \hline & \multicolumn{7}{c}{ Speaker } \\
& SL & HS & ER & LB & MW & AB & Total \\
\hline Mean & $41 \%$ & $49 \%$ & $49 \%$ & $67 \%$ & $46 \%$ & $49 \%$ & $\mathbf{5 0 \%}$ \\
Range (\%) & $19-68$ & $25-70$ & $17-80$ & $21-65$ & $22-61$ & $17-75$ & $\mathbf{1 7 - 8 0}$ \\
SD & 10.7 & 10.7 & 17.1 & 13.4 & 9.3 & 14.2 & $\mathbf{1 5 . 0}$ \\
n & 38 & 46 & 47 & 35 & 28 & 23 & $\mathbf{2 1 7}$ \\
\hline \hline
\end{tabular}

(3) Results for peak location as a proportion of rhyme duration.

\begin{tabular}{cccccccc}
\hline \hline & \multicolumn{7}{c}{ Speaker } \\
SL & HS & ER & LB & MW & AB & Total \\
\hline Mean & $23 \%$ & $32 \%$ & $25 \%$ & $26 \%$ & $30 \%$ & $24 \%$ & $\mathbf{2 8 \%}$ \\
Range (\%) & $11-37$ & $21-53$ & $12-52$ & $16-45$ & $19-50$ & $11-35$ & $\mathbf{1 1 - 5 3}$ \\
SD & 7.8 & 7.8 & 9.1 & 6.7 & 8.1 & 7.0 & $\mathbf{8 . 6}$ \\
n & 38 & 46 & 47 & 35 & 28 & 23 & $\mathbf{2 1 7}$ \\
\hline \hline
\end{tabular}

Examining the means for syllable duration, it appears that the peak aligns, on average, at the middle of the syllable - an intuitively appealing result, especially when compared to the alignment based on rhyme duration, which seems rather arbitrary at $28 \%$. However, in looking at the overall ranges for each syllable type, we see that the rhyme proportions have a range from $11 \%$ to $53 \%$, which is a rather tighter range than that for the syllable proportions of $17 \%$ to $80 \%$. This indicates that the rhyme provides a tighter fit for the location of the peak. This conclusion is supported by examining the standard deviations of the two 


\section{Tolowa Peak Pitch Alignment}

proportions. The alignment by syllable duration has a standard deviation $[\mathrm{SD}=15.0]$ almost twice as large as the standard deviation of the alignment-byrhyme group $[\mathrm{SD}=8.6]$. An F-test on the variance of the syllable duration group $\left[\sigma^{2}=225.39\right]$ and the rhyme duration group $\left[\sigma^{2}=73.12\right]$ was computed which indicated that peak delay calculated as a proportion of the duration of the rhyme was significantly less variable than the proportion computed based on the duration of the entire syllable $[F(216,216)=3.08, p<.001]$.

Due to the fact that there is less variability in the alignment of the peak pitch in terms of the proportion of the rhyme than of the syllable, it appears that the rhyme is the domain of peak alignment in Tolowa. This mirrors the finding of Silverman and Pierrehumbert (1990) for English and Arvaniti et al. (1998) for Modern Greek but stands in contrast the findings of Prieto et al. (1995) for Mexican Spanish.

\subsection{Effects of Segmental Structure}

We now turn to an examination of the second question raised above: is the alignment of the peak affected by the structure of the syllable with which it is aligned? Of specific interest was the role that coda consonants play in alignment of the peak, as the peaks in CVN and CVO syllables often occurred quite close to the end of the vowel, and thus just before the beginning of the coda consonant. Because the above results demonstrated that a proportion based on rhyme duration is the best predictor of peak delay, only this proportion will be considered in the analysis that follows. The same tokens used above are also used here; however, tokens were separated into different groups based on syllable type (CVV, CVN, \& CVO). Mean proportions for each type of syllable by speaker are presented in Table (4).

(4) Means, standard deviations, and number of tokens for peak alignment by syllable type and speaker.

\begin{tabular}{|c|c|c|c|c|c|c|c|c|}
\hline \multicolumn{9}{|c|}{ Speaker } \\
\hline & & SL & HS & ER & LB & MW & $\mathbf{A B}$ & Total \\
\hline \multirow{3}{*}{ | } & M & $28 \%$ & $38 \%$ & $31 \%$ & $28 \%$ & $35 \%$ & $26 \%$ & $31 \%$ \\
\hline & SD & 6.0 & 11.0 & 9.6 & 6.1 & 6.2 & 7.0 & 8.5 \\
\hline & $\mathbf{n}$ & 16 & 11 & 18 & 16 & 7 & 8 & 76 \\
\hline \multirow{3}{*}{ 至 } & M & $21 \%$ & $34 \%$ & $32 \%$ & $28 \%$ & $28 \%$ & $27 \%$ & $28 \%$ \\
\hline & SD & 7.5 & 6.1 & 2.3 & 8.2 & 3.0 & 4.1 & 6.4 \\
\hline & $\mathbf{n}$ & 5 & 4 & 3 & 7 & 7 & 7 & 33 \\
\hline \multirow{3}{*}{$\begin{array}{l}\text { 이 } \\
\text { 己े }\end{array}$} & M & $19 \%$ & $30 \%$ & $20 \%$ & $21 \%$ & $29 \%$ & $18 \%$ & $24 \%$ \\
\hline & SD & 7.6 & 5.6 & 5.4 & 4.0 & 10.1 & 5.8 & 8.1 \\
\hline & $\mathbf{n}$ & 17 & 31 & 26 & 12 & 14 & 8 & 108 \\
\hline
\end{tabular}




\section{Christopher S. Doty and Susan G. Guion}

In examining the peak alignment data presented in Table (4), CVV and CVN syllables appear relatively similar, with means of $31 \%$ and $29 \%$, respectively, while peaks in CVO syllables occur slightly earlier, at $24 \%$ of the length of the rhyme. To test whether these differences were significant, the data were submitted to a two-way GLM ANOVA, with speaker (six levels) and syllable type (three levels) as independent factors and proportion of the rhyme duration at which the peak occurred as the dependent variable. The effect of both speaker $[F(5,199)=9.332, p<.001]$ and syllable type $[F(2,199)=27.034, p<.001]$ were significant. The interaction between the two factors, however, was not significant $[p=.399]$. This indicates that speakers were relatively consistent in their differentiation of the syllable types in terms of peak alignment.

Tukey's HSD tests were conducted to determine which levels of syllable type were significantly different from each other. The tests indicated that CVO syllables $[\mathrm{M}=24 \%, \mathrm{SD}=8.1]$ had a peak which occurred significantly earlier than both CVV syllables [M=31\%, $\mathrm{SD}=8.5]$ and $\mathrm{CVN}$ syllables $[\mathrm{M}=28 \%, \mathrm{SD}=6.4]$, $[p=.001$ and .012, respectively]. The difference between CVV and CVN syllables was not significant [ $p=.154]$.

Based on these results, it appears that the type of coda in a given syllable affects the alignment of the pitch-accent peak, with peaks in CVO syllables occurring earlier than peaks in syllables with other types of codas. Given that all stops and fricatives in this position are voiceless in Tolowa, one might suspect that this earlier alignment of the peak is related to the fact that the peak cannot be realized during the voiceless portion of the rhyme, and the high target is thus moved closer to the beginning of the syllable. This sort of time pressure would not be a problem in CVV and CVN syllables, as voicing continues throughout the rhyme. A look at the mean durations of the rise in all syllable types-measured from vowel onset to the peak-indicates that CVN syllables [M=85 ms], although slightly shorter than CVV syllables [M=93 $\mathrm{ms}]$, are still longer than CVO syllables $[\mathrm{M}=75 \mathrm{~ms}]$. It follows, then, that if this time pressure has indeed forced the high target at the peak to be realized earlier in CVO syllables, then we also ought to see a steeper slope to the rise in CVO syllables than in CVV or CVN syllables, as the rise now has less time to be completed (see below).

\subsection{Slope of the Rise}

Because the peak in CVO syllables occurs significantly earlier than in CVV or CVN syllables, the autosegmental framework predicts that the F0 contour in CVO syllables will have a steeper slope. This is due to the fact that the distance between the low target at the beginning of the syllable and the high target during the rhyme has been reduced. Thus, if the slope of a pitch contour is simply an extrapolation between two targets, we would expect the slope to become steeper as the distance between the two targets decreases (as has been found in several previous studies, e.g., Prieto et al. 1995).

In order to test this prediction, a measurement of the slope was calculated as follows. The duration of the rise in milliseconds (time of high minus time of low), 


\section{Tolowa Peak Pitch Alignment}

and the difference between the peak and the low (in $\mathrm{Hz}$ ) was calculated. From these two numbers, the slope of the rise in $\mathrm{F} 0$ was calculated $(\mathrm{Hz} / \mathrm{ms})$. A slope of, for example, .25 represents a one-quarter Hertz rise in pitch for every millisecond that the contour lasts. Higher numbers thus indicate a steeper slope.

Means and standard deviations for each syllable type by speaker are shown in Table (5). As is clear from the table, CVV syllables appear to have a slope that, with a rise of .241 Hz/ms, is less steep than both CVN and CVO syllables, with slopes of .341 and $.299 \mathrm{~Hz} / \mathrm{ms}$, respectively.

(5) Slope of the F0 contour by speaker and syllable type.

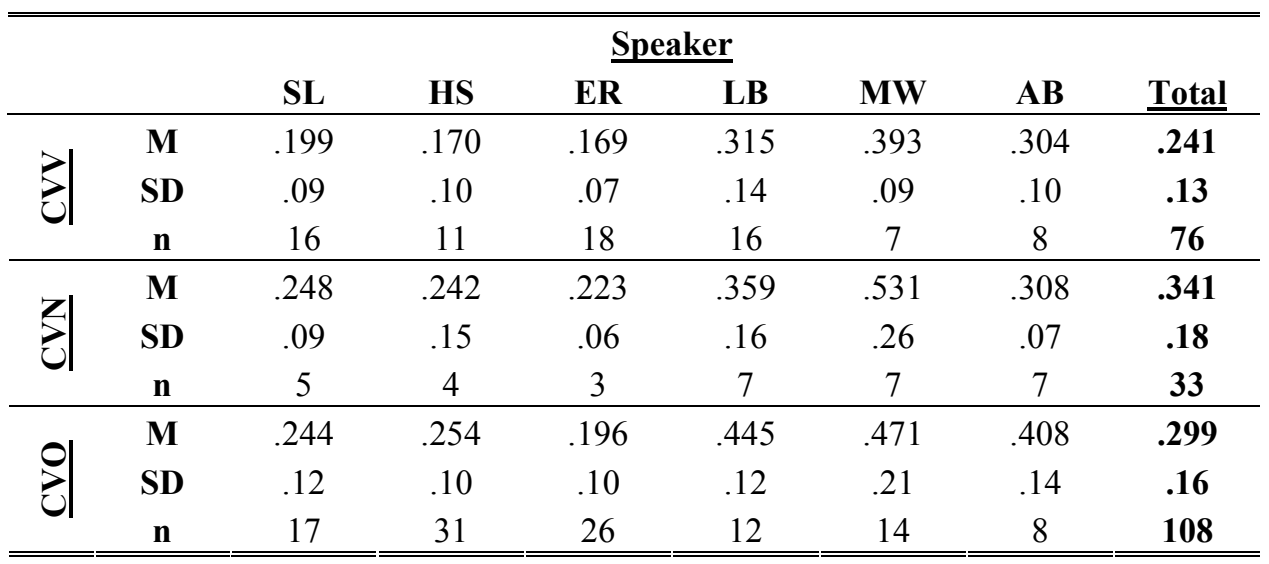

These data were submitted to a GLM ANOVA with speaker and syllable type as independent factors and the slope of the pitch contour from vowel onset to peak pitch as the dependent variable. Results of the ANOVA indicated that the effects of both speaker $[F(5,199)=18.010, p<.001]$ and syllable type $[F(2,199)=7.715$, $p=.001]$ were significant. The interaction of speaker and syllable type was not significant $[p=.658]$, indicating that speakers were consistent in their production of the slope of the contour by syllable type.

Tukey's HSD pair-wise comparisons were conducted on the slope measurements to determine which syllable types were significantly different from each other. $\mathrm{CVV}$ syllables $[\mathrm{M}=.241, \mathrm{SD}=.13]$ were found to have slopes which were significantly more shallow than the slopes of both $\mathrm{CVN}$ syllables $[\mathrm{M}=.341$, $\mathrm{SD}=.17, p<.001]$ and $\mathrm{CVO}$ syllables $[\mathrm{M}=.299, \mathrm{SD}=.16, p=.005]$. The difference between CVN and CVO syllables was not significant [ $p=.208]$.

Although CVV syllables are realized with a more shallow slope than CVO syllables as predicted by autosegmental theory, CVN syllables pattern not with CVV syllables in terms of their alignment, but rather with CVO syllables. This is despite the fact that previous findings indicated that CVV and CVN syllables clearly pattern together in terms of the peak's alignment within the rhyme. This discrepancy is represented graphically in Figure (2). 


\section{Christopher S. Doty and Susan G. Guion}

(2) Grouping of CVV, CVN and CVO syllable types based on two criteria: peak alignment and slope of rise.

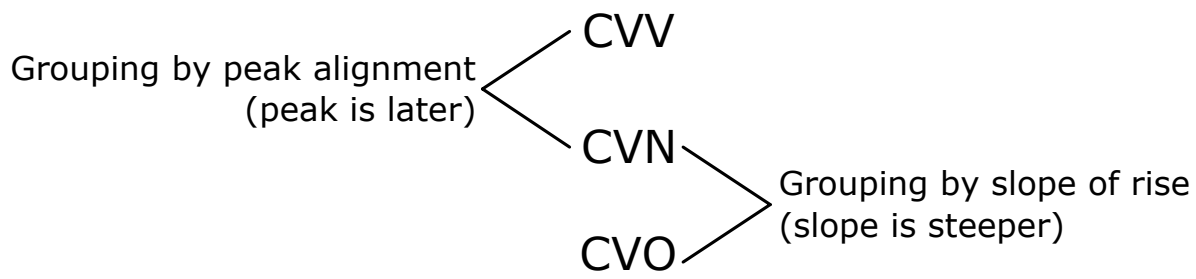

One possible explanation presents itself based on the mean durations of the rise presented above in Section 2.2. Recall that these means showed that CVN syllables were closer to CVV syllables than CVO syllables in terms of the length of the rise. However, it now seems that, despite the fact that CVN syllables are closer to CVV syllables in terms of the duration of the rise $[\mathrm{M}=85 \mathrm{~ms}$ and $\mathrm{M}=93$ $\mathrm{ms}$, respectively] than to CVO syllables $[\mathrm{M}=75 \mathrm{~ms}]$, the shorter duration of the rise in CVN syllables is sufficient to cause an increase in the slope of the rise such that CNV syllables pattern with CVO syllables.

However, this still does not explain why the peak alignment in CVN syllables matches that in CVV syllables. If the duration of the rise is shorter, it seems that we ought to have seen peaks which were earlier as well, but this was not the case. We now turn to the General Discussion \& Conclusion, where we will offer one speculative possibility to explain these data further.

\section{General Discussion \& Conclusion}

The preceding investigation has provided a first look at peak pitch-accent alignment in stressed Tolowa syllables. To summarize, the results were as follows. First, the rhyme rather than the syllable was shown to be the domain for peak pitch alignment, with a proportion based on the duration of the rhyme providing the best description of the alignment of the peak. Second, it was demonstrated that peaks align significantly earlier in CVO syllables than in CVV or CVN syllables. It was proposed that this earlier alignment is the result of time pressure to realize the high target at the peak prior to the voiceless obstruent coda. Finally, it was shown that the slope of the rise in CVO and CVN syllables is significantly steeper than the rise in CVV syllables. These findings are presented in Table (6).

Although some questions about peak pitch accent alignment in Tolowa have been answered, additional questions have been raised by apparently conflicting results. Given than CVN syllables have a peak which is aligned like that of CVV syllables, we would expect these two syllable types to have similar slopes; there thus seems to be no obvious explanation for the fact that CVN syllables have a steeper slope than CVV syllables and pattern like CVO syllables. Although the durations of the rises may have given us a hint as to why the slope is steeper in 


\section{Tolowa Peak Pitch Alignment}

CVN syllables, it remains unclear why the peaks in these cases would then align like CVV syllables.

(6) Summary of the results of the present study.

\begin{tabular}{c}
\hline \hline Findings \\
\hline Rhyme is domain of peak alignment \\
Peaks align earlier in CVO than CVV or CVN \\
Slopes in CVN and CVO steeper than in CVV \\
\hline \hline
\end{tabular}

One possible explanation for this result is that the presence of any coda consonant is taken into account when calculating where to align the peak pitch target; that is to say that in both CVN and CVO syllables, there may be a certain pressure to realize the pitch target before the coda consonant begins-regardless of whether that consonant is voiced or voiceless - which could cause the peak to be aligned earlier. This could then lead to both CVN and CVO syllables having a steeper slope. However, this account would not then be able to explain why the peaks of CVN syllables actually align like the peaks of CVV syllables, unless the voicing of the nasal coda allows a certain amount of flexibility in aligning the peak, which is why it is seen to occur later than it might otherwise. This may be somewhat supported by the fact that the duration of the rise in CVN syllables is intermediate between CVV and CVN syllables. Further investigation is warranted in order to determine if this explanation might help to account for these disparate data.

One additional point ought to be considered in a similar vein. Given that the alignment of the peaks in CVO and CVN syllables appears to be close to the end of the vowel, one might be tempted to analyze this as the location with which the peak is aligned. A problem with this analysis becomes apparent when CVV syllables are considered. However, recall that all open syllables are phonologically lengthened in Tolowa. It might be the case that the alignment of the peak in CVV syllables is a historical remnant of a time prior to this lengthening rule, when the peak perhaps aligned with the end of the vowel in *CV syllables. Further comparative work would be needed to substantiate this hypothesis.

\section{References}

Arvaniti, Amalia, Robert D. Ladd, and Ineke Mennen. 1998. Stability of tonal alignment: the case of Greek prenuclear accents. Journal of Phonetics 26:325.

Boersma, Paul, and David Weenik. 2006. Praat: doing phonetics by computer. 


\section{Christopher S. Doty and Susan G. Guion}

Bommelyn, Loren. 2006. Taa-laa-waa Dee-ni' Wee-ya'. Smith River, CA: Smith River Rancheria.

Bright, Jane O. 1964. The Phonology of Smith River Athapaskan (Tolowa). International Journal of American Linguistics 30:101-107.

Ladd, Robert D. 1996. Intonational Phonology. Cambridge: Cambridge University Press.

Ladd, Robert D., Ineke Mennen, and Astrid Schepman. 2000. Phonological conditioning of peak alignment in rising pitch accents in Dutch. Journal of the Acoustical Society of America 107:2685-2696.

Prieto, Pilar, Jan van Santen and Julie Hirschberg. 1995. Tonal alignment patterns in Spanish. Journal of Phonetics 23:429-451.

Silverman, Kim E. A., and Janet B. Pierrehumbert. 1990. The timing of prenuclear high accents in English. In J. Kingston and M. E. Beckman, eds., Papers in Laboratory Phonology 1. New York: Cambridge University Press.

Tuttle, Siri G. 1990. Stress and Vowel Length in Tolowa. M.A. thesis, University of Washington.

Christopher S. Doty

Department of Linguistics

1290 University of Oregon

Eugene, OR 97403-1290

cdoty@uoregon.edu 